\title{
Study on the Modification of Humic Acid and Its Adsorption to Methylene Blue
}

\author{
Chen Rongping, Zhang Zishuai, Wu Dinggui \\ College of Biology and Environment, Nanjing Forestry University, Nanjing 210037, P.R. China
}

Keywords: Humic acid; Adsorption; Methylene blue

\begin{abstract}
Humic acid insoluble(IHA) was prepared via the method of immersing in calcium salt. The structure and properties of IHA were characterized by FTIR and Zeta. The effect factors of adsorption such as $\mathrm{pH}$, temperature and time were studied. Adsorptive removal of methylene blue (MB) from aqueous solution using IHA was systematically studied. The characterization results of adsorbent showed that: the zeta potential is negative in the $\mathrm{pH}$ range of $1 \sim 9$ due to the carboxyl groups on the IHA. The experimental results of adsorption showed that the adsorption capacity was increasing with the increase of the $\mathrm{pH}$. The adsorption equilibrium study indicated that the adsorptions of MB were monolayer and spontaneous processes. The adsorption kinetics followed pseudo-second-order equation. The activation energy value indicated that the adsorption was a physical process. Furthermore, IHA could be easily regenerated and reused with little loss of adsorption capacity.
\end{abstract}

\section{Introduction}

Dye is a class of pollutant that is found in the effluents of textile, paper, plastic, food and cosmetic industries [1]. The high chromaticity impedes light penetration and thus prohibits aquatic photosynthesis. Considering the hazard of dyes, study on the removal of dyes from water body is of great environmental significance at present [1-3].

Nowadays, the most widely used water-treating techniques are flocculation, adsorption, catalyzed degradation, filtration, electrochemical methods and biological methods [4-8]. Among them, adsorption is widely applied in further remediation of wastewater due to its high removal efficiency and process convenience [9]. In recent years, numerous studies were carried out concerning adsorptive water treatment using green materials, especially natural polymers, since they are inexpensive, eco-friendly and tunable [10].

Humic acid (HA) is a class of natural organic polymers which originates from the decomposition of plants and animal residues. HA exhibits fine reaction activity for bearing abundant functional groups such as carboxyl, phenolic, carbonyl and amino groups. These groups make it easy for HA to bind cationic species from aqueous solutions [11]. But HA can't be used as an adsorbent directly for its water solubility.

In this work, we prepared a green adsorbent based on humic acid by immersing HA in calcium salt. Physiochemical characterizations of IHA were performed using modern analytical methods. Adsorption behaviors of IHA, including the effect of the initial solution $\mathrm{pH}$, adsorption kinetics and isothermal adsorption equilibrium, were all investigated. In addition, Recycling and reuse of the disused adsorbents were also carried out for evaluating its application potential.

\section{Experimental}

Humic Acid was purchased from Aladdin Industrial Corporation. Calcium chloride and hydrochioric acid were purchased from Sinopharm Chemical Reagent Co. Ltd. All the reagents used in this work are A.R. grade. Deionized water was used in all experiments.

$5 \mathrm{~g}$ of humic acid was immersing in $100 \mathrm{ml}$ of calcium chloride $(1 \mathrm{~mol} / \mathrm{l})$ solution for $2 \mathrm{~h}$. Collected from the solution by fitration and washed to remove excessive Calcium chloride by Hydrochloric acid, then washed to neutral with Deionized water. Afterwards the black precipitates were freezing dried for further use. 
IHA underwent multiple characterizations: Fourier transform infrared spectroscopy (FTIR, on Avatar360; Nicolet Co.; USA); Zeta potential analysis (on Nano ZS90; Co.; UK);

The initial $\mathrm{pH}$ ranges of the solutions were 2.0-10.0 for MB. The initial concentration of MB was $100 \mathrm{mg} / \mathrm{L}$, based on adsorption isotherms. $0.05 \mathrm{~g}$ (dry weight) of IHA was immerged in $50 \mathrm{~mL}$ of MB solution and stirred for $24 \mathrm{~h}$ to reach the equilibrium.

Adsorption capacity in subsequent experiments, qe (mg/g), was calculated according to the following equation unless noted:

$$
q_{e}=\frac{\left(C_{0}-C_{e}\right) V}{m}
$$

Where $C_{0}$ and $C_{e}(\mathrm{mg} / \mathrm{L})$ are the initial and equilibrium $\mathrm{MB}$ concentrations in solution, respectively; $V(\mathrm{~L})$ is the volume of solution; $m(\mathrm{~g})$ is the weight of dry adsorbents.

Kinetic adsorption experiments were performed at $293 \mathrm{~K}$ and initial $\mathrm{pH}$ 7.0. The initial concentration of MB solution was the same as in Section 2.4.1. $0.5 \mathrm{~g}$ of adsorbent was immerged into $500 \mathrm{~mL}$ MB solution under continuous agitation. Then, $1 \mathrm{~mL}$ of sample solutions was taken out at certain time intervals to measure MB concentration. Meanwhile, equivalent volume of water with $\mathrm{pH} 7.0$ was added into the bulk solutions to maintain the solution volume.

The dye uptake $q\left(t_{\mathrm{i}}\right)(\mathrm{mg} / \mathrm{g})$ at time $t_{\mathrm{i}}$ was calculated using the following equation:

$$
q\left(t_{i}\right)=\frac{\left(C_{0}-C_{t_{i}}\right) V_{o}-\sum_{2}^{i-1} C_{t_{i-1}} V_{a}}{m}
$$

Where $\mathrm{C} 0$ and $\mathrm{Cti}(\mathrm{mg} / \mathrm{L})$ are the initial dye concentrations and the concentrations at the time ti, respectively. V0 and Va (L) are the volume of the mixed solution and the volume of the sample solution taken out at ti for analysis, respectively. In this case, Va equals to $1.0 \mathrm{~mL}$ and $\mathrm{m}(\mathrm{g})$ represents the weight of the adsorbent.

Adsorption equilibrium study was also conducted at $293 \mathrm{~K}$ and initial solution $\mathrm{pH} 7.0$ with the concentrations of MB solutions ranged from 10 to $200 \mathrm{mg} / \mathrm{L}$. $0.05 \mathrm{~g}$ of MHA adsorbent was immerged into $50 \mathrm{~mL}$ of $\mathrm{MB}$ dye solution under continuous stirring. The same method as mentioned in the previous section was employed to analyze the initial and final MB concentrations.

Regeneration and reuse of adsorbents is of great importance in practical applications. The MB loaded IHA was recovered using $0.1 \mathrm{~mol} / \mathrm{L}$ HNO3 solution, and then collected from the solutions by filtration, washed with distilled water. The regenerated adsorbents were reused in the next cycle of adsorption experiments. The experiment was conducted for five cycles.

\section{Results and discussion}

FTIR spectra of IHA are shown in Fig.1. The band at $1650 \mathrm{~cm}-1$ of IHA is ascribed to the $\mathrm{C}=\mathrm{O}$ stretching band [11]. The band at $1400 \mathrm{~cm}-1$ can be ascribed to $\mathrm{CH} 2$ scissoring [11]. The band at $1050 \mathrm{~cm}-1$ is the C-O stretching of COO-.



Fig.1 FTIR spectra of IHA 
Zeta potentials of the IHA were measured at different $\mathrm{pH}$ and shown in Fig.2. On Fig.2, it is observed that the zeta potential of IHA is negative in the $\mathrm{pH}$ range of 1.0 9.0. The zeta potential plot of IHA suggests MHA is negatively charged at the environment of $\mathrm{pH}$ 1.5 9.2, which make it is beneficial for the adsorption of cationic species.

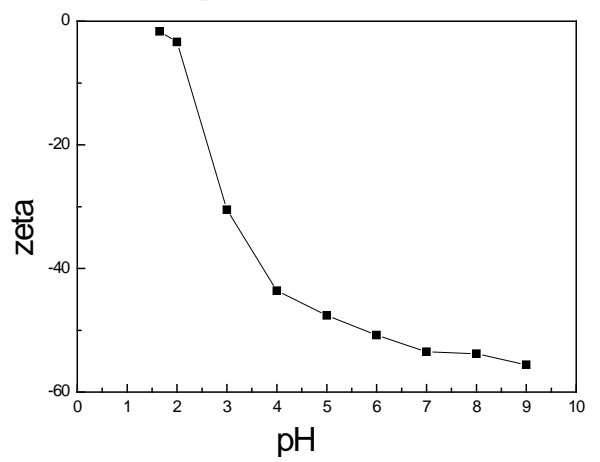

Fig.2 Zeta potential curve of IHA at different initial solution $\mathrm{pH}$.

The plot of adsorption capacity versus initial $\mathrm{pH}$ is shown in Fig.3. The upper limit of studied $\mathrm{pH}$ is set at 10 since at $\mathrm{pH}$ higher than the value, $\mathrm{MB}$ will precipitate in solution, making results invalid [12]. As is shown in Fig.3, the adsorption of MB on IHA is greatly influenced by initial solution $\mathrm{pH}$. IHA exhibits efficient $\mathrm{MB}$ removal in the initial solution $\mathrm{pH}$ range of 6-10, where the adsorption capacity is about $50 \mathrm{mg} / \mathrm{g}$. The adsorption to $\mathrm{MB}$ is weak in solutions when initial $\mathrm{pH}$ is lower than 3 , which is due to the protonation of the anionic groups on IHA. When the $\mathrm{pH}$ is higher than 3 , the surface of IHA becomes negatively charged, as the surface carboxyl groups deprotonate. The negatively charged IHA can better react with cationic MB through electrostatic attraction.

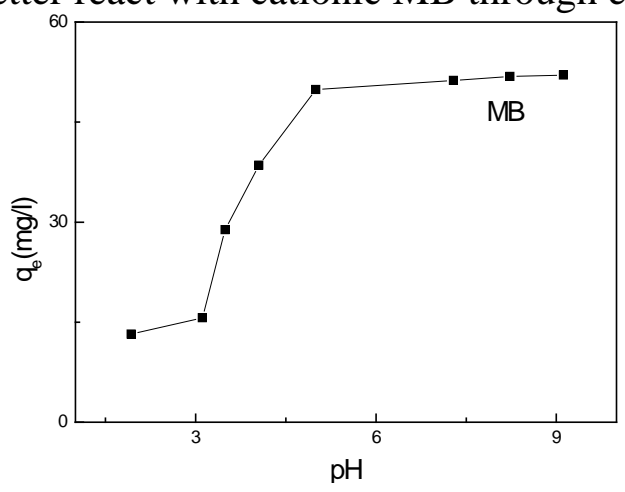

Fig.3. Effect of initial solution $\mathrm{pH}$ on MB adsorption onto IHA

The kinetic adsorption results at three temperatures were shown in Fig.4. It is observed that the adsorption equilibrium of IHA for MB is achieved about $5 \mathrm{~h}$ and the adsorption capacity is about 50 $\mathrm{mg} / \mathrm{g}$ at $293 \mathrm{~K}$.

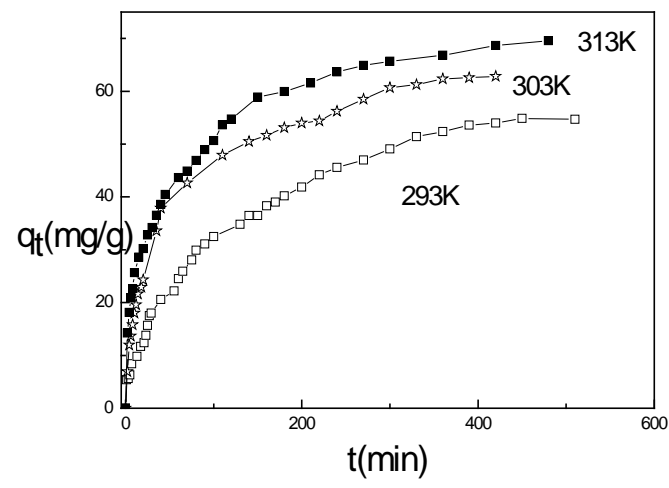

Fig.4 Adsorption kinetics of MB onto IHA

To further study the adsorption mechanism, three kinetic models are used to simulate the kinetic 
data, namely pseudo-first order, pseudo-second order and intraparticle diffusion models.

The pseudo-first-order kinetic model is expressed as the following equation:

$$
\ln \left(q_{e}-q_{t}\right)=\ln q_{e}-k_{1} t
$$

Where $\mathrm{k} 1$ (min-1) is the rate constant of pseudo-first-order adsorption; qe and qt (mg/g) are the amount of MB absorbed onto adsorbents at equilibrium and at time $\mathrm{t}(\mathrm{min})$, respectively.

The pseudo-second-order kinetic model can be expressed Eq. (4):

$$
\frac{t}{q_{t}}=\frac{1}{k_{2} q_{e}^{2}}+\frac{t}{q_{e}}
$$

Where qe and qt (mg/g) are the amounts of MB adsorbed onto adsorbents at equilibrium and at time $\mathrm{t}(\mathrm{min})$, respectively; $\mathrm{k} 2$ (g/ (mg min)) is the rate constant of pseudo-second-order model.

Intraparticle diffusion model suggests that intraparticle diffusion is the rate-limiting step in the adsorption. The model can be described as the following equation:

$$
q_{t}=k_{p} t^{0.5}+c
$$

Where $\mathrm{kp}(\mathrm{mg} /(\mathrm{g} \cdot \mathrm{min} 0.5))$ is the intraparticle diffusion rate constant, and $\mathrm{c}(\mathrm{mg} / \mathrm{g})$ is the intercept of this equation.

The experimental data are fitted according to these kinetics models, the parameters are listed in Table 1. As the correlation coefficient (R2) of pseudo-second order model is much closer to 1.0 than other employed kinetic models, and the theoretical qe values are also closer to the experimental ones, which indicates that the pseudo-second order kinetic model is the most appropriate to interpret

\begin{tabular}{|c|c|c|c|c|c|c|c|c|c|c|}
\hline \multirow[b]{2}{*}{$T(\mathrm{~K})$} & \multicolumn{4}{|c|}{$\begin{array}{l}\text { Pseudo first-order } \\
\text { model }\end{array}$} & \multicolumn{3}{|c|}{ Pseudo second-order model } & \multicolumn{3}{|c|}{$\begin{array}{c}\text { Intraparticle diffusion } \\
\text { model }\end{array}$} \\
\hline & $\begin{array}{c}q_{\mathrm{e}, \exp } \\
(\mathrm{mg} / \mathrm{g})\end{array}$ & $\begin{array}{c}k_{1} \\
\left(\min ^{-1}\right)\end{array}$ & $\begin{array}{c}q_{\mathrm{e}, \mathrm{cal}} \\
(\mathrm{mg} / \mathrm{g})\end{array}$ & $R^{2}$ & $\begin{array}{c}k_{2} \\
* 10^{4}(\mathrm{~g} / \mathrm{mg} \\
\mathrm{min})\end{array}$ & $\begin{array}{c}q_{\mathrm{e}, \mathrm{cal}} \\
(\mathrm{mg} / \mathrm{g})\end{array}$ & $R^{2}$ & $\begin{array}{c}k_{\mathrm{p}}(\mathrm{mg} / \mathrm{g} \\
\left.\min ^{0.5}\right)\end{array}$ & $\begin{array}{c}C \\
(\mathrm{mg} / \mathrm{g})\end{array}$ & $R^{2}$ \\
\hline 293 & 54.69 & 0.009 & 59.15 & 0.925 & 2.70 & 55.56 & 0.991 & 2.60 & 3.43 & 0.978 \\
\hline 303 & 63.12 & 0.012 & 57.28 & 0.934 & 4.67 & 66.67 & 0.994 & 2.92 & 10.93 & 0.945 \\
\hline 313 & 70.65 & 0.008 & 47.94 & 0.983 & 4.23 & 76.92 & 0.994 & 2.76 & 18.95 & 0.931 \\
\hline
\end{tabular}
the adsorption process.

Tab.1. Kinetic parameters for MB onto IHA

To further analyze the kinetic behavior, the activation energy of the adsorption was calculated based on Arrhenius equation, which is expressed as the following equation [12]:

$$
\ln k_{2}=-\frac{E_{a}}{R T}+\ln (A)
$$

Where $\mathrm{k} 2$ is the rate constant of pseudo-second-order adsorption $(\mathrm{g} /(\mathrm{mg} \cdot \mathrm{min}))$, as the adsorption kinetics is well described by this model; Ea is the activation energy of adsorption $(\mathrm{kJ} / \mathrm{mol})$; $A$ is the Arrhenius factor; $\mathrm{R}$ is the universal gas constant $(8.314 \mathrm{~J} /(\mathrm{mol} \cdot \mathrm{K}))$; $\mathrm{T}$ is the temperature $(\mathrm{K})$.

A plot of lnk2 versus 1/T yields a straight line, with slope - Ea/R. The value of Ea can be used to differentiate physical and chemical adsorptions. Physical adsorption can achieve equilibrium quickly, and the energy requirement is usually small, ranging from 5 to $30 \mathrm{~kJ} / \mathrm{mol}$ [12]. Whereas chemical adsorption involves stronger interactions and requires higher activation energy, ranging from 30 to $800 \mathrm{~kJ} / \mathrm{mol}$ [12]. The value of Ea based on calculation is $17.33 \mathrm{~kJ} / \mathrm{mol}$, indicating that the adsorption of MB onto IHA is a physical adsorption process and is consistent with the 
experimental results.

Adsorption isotherms of MB onto IHA at different temperatures are shown in Fig.5. It is found that the amount of adsorbed MB increase with the increase of the reaction temperature, which indicates the adsorption is more favorable at higher temperature. To assess MB adsorption behavior on the IHA, the data were analyzed based on Langmuir and Freundlich isothermal models.

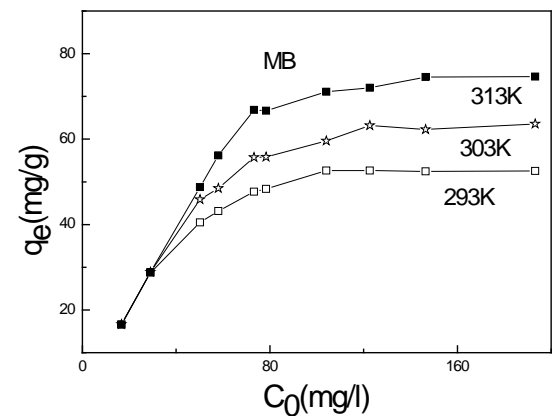

Fig.5. Adsorption isotherms of MB onto IHA at various temperatures

Langmuir model is a commonly used model based on the assumption of a monolayer adsorption on a homogeneous surface ${ }^{[11]}$. The equation is expressed in Eq. (7):

$$
\frac{C_{e}}{q_{e}}=\frac{1}{q_{m} b}+\frac{C_{e}}{q_{m}}
$$

Where qe $(\mathrm{mg} / \mathrm{g})$ is the amount of $\mathrm{MB}$ adsorbed at equilibrium, Ce $(\mathrm{mg} / \mathrm{L})$ is the $\mathrm{MB}$ concentration at equilibrium, qm (mg/g) is the maximum adsorption capacity when the adsorbent is fully covered, and b $(\mathrm{L} / \mathrm{mg})$ is the Langmuir adsorption constant which relates to the adsorption energy.

Freundlich isotherm model assumes multilayer adsorption with sites of exponentially distributed adsorption energies and is expressed in Eq. (8).

$$
\ln \left(q_{e}\right)=\ln \left(K_{f}\right)+\frac{1}{n} \ln \left(C_{e}\right)
$$

Where $\mathrm{Kf}$ is the Freundlich isotherm constant and $\mathrm{n}$ (dimensionless) is the heterogeneity factor. Ce is the equilibrium concentration (mg/L); qe is the amount of $\mathrm{MB}$ adsorbed at equilibrium (mg/g).

The simulation results based on the two models were listed in Tab. 2. According to the Tab. 2, the correlation coefficients (R2) of the linear form for Langmuir model are higher than those of Freundlich at all the temperatures. Thus the isothermal adsorption behavior of MB onto IHA follows Langmuir equation, indicating a monolayer adsorption of MB occurring on a homogeneous surface.

Tab.2. Isothermal parameters for the adsorption of MB onto MHA based on Langmuir and Freundlich models

\begin{tabular}{ccccc}
\hline \multirow{2}{*}{ Model } & \multirow{2}{*}{ Parameter } & \multicolumn{3}{c}{ Temperature(K) } \\
\cline { 2 - 5 } & & 293 & 303 & 313 \\
\hline \multirow{3}{*}{ Langmuir } & $q_{m}(\mathrm{mg} / \mathrm{g})$ & 55.56 & 66.67 & 76.92 \\
& $b(\mathrm{l} / \mathrm{mg})$ & 0.17 & 0.14 & 0.12 \\
& $R^{2}$ & 0.999 & 0.999 & 0.999 \\
\multirow{2}{*}{ Freundlich } & $K_{f}$ & 28.11 & 32.79 & 37.11 \\
& $n$ & 6.85 & 6.29 & 5.41 \\
& $R^{2}$ & 0.904 & 0.928 & 0.859 \\
\hline
\end{tabular}

To further study the adsorption mechanism, thermodynamic parameters are calculated based on the following equations:

$$
\Delta G=-R T \ln b
$$




$$
\Delta G=\Delta H-T \Delta S
$$

Where $b$ is the thermodynamic distribution coefficient obtained from Langmuir model $(\mathrm{L} / \mathrm{mol})$; $\mathrm{R}$ is the universal gas constant $(8.314 \mathrm{~J} /(\mathrm{mol} \cdot \mathrm{K}))$; $\mathrm{T}$ is the temperature $(\mathrm{K}) . \Delta \mathrm{G}(\mathrm{kJ} / \mathrm{mol})$ is Gibbs free energy; $\Delta \mathrm{H}(\mathrm{kJ} / \mathrm{mol})$ is the enthalpy change and $\Delta S(\mathrm{~J} /(\mathrm{mol} \cdot \mathrm{K}))$ is the entropy change.

The thermodynamic parameters obtained based on Eq. (9) and (10) are listed in Table 3. The values of $\triangle G$ at all the temperatures are negative, indicating spontaneous adsorptions at these temperatures. The value of $\Delta H$ of $M B$ is negative while $\Delta S$ is positive, suggesting that the adsorption process is an exothermal process with entropy increase.

Tab.3. Thermodynamic parameters for the adsorption of MB onto IHA

\begin{tabular}{ccccc}
\hline$\Delta H(\mathrm{~kJ} / \mathrm{mol})$ & $\Delta S(\mathrm{~J} / \mathrm{mol} \mathrm{K})$ & \multicolumn{3}{c}{$\Delta G(\mathrm{~kJ} / \mathrm{mol})$} \\
\hline \multirow{2}{*}{42.39} & \multirow{2}{*}{3.15} & $293 \mathrm{~K}$ & $303 \mathrm{~K}$ & 313 \\
\cline { 2 - 5 } & & -26.51 & -26.96 & -27.48 \\
\hline
\end{tabular}

The regeneration results are shown in Fig.6. As shown in Fig.6, the adsorption capacity of MB only experienced a very slight drop after five cycles. It suggested that the reusability of IHA is fine, which is satisfactory for practical applications.

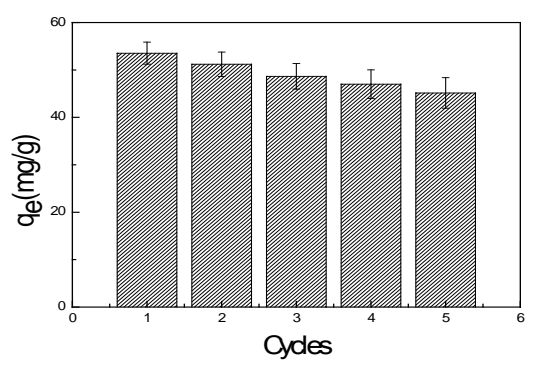

Fig.6. Variation on adsorption capacity in regeneration cycles of IHA

\section{Conclusion}

In this work, a bio-degradable adsorbent, insoluble humic acid (IHA) was prepared. IHA shows fine removal efficiency for MB in aqueous solution. The zeta potential plot of IHA suggests IHA is negatively charged at the environment of $\mathrm{pH}$ 1.0 9.0, which make it is beneficial for the adsorption of cationic species.

The adsorption equilibrium of IHA for adsorption of MB is achieved in $5 \mathrm{~h}$ and the adsorption kinetic process can be followed by pseudo-second-order model. The equilibrium adsorption data is in agreement with Langmuir isotherm model with a maximum adsorption amount of about $50 \mathrm{mg} / \mathrm{g}$ at $293 \mathrm{~K}$. The value of activation energy (Ea) indicates that the adsorption of MB onto IHA is a physical adsorption process. The value of Gibbs free energy $(\triangle G)$ is negative, indicating that the adsorption of MB onto IHA is spontaneous and thermodynamically favorable. Moreover, the adsorbents can be readily regenerated and efficiently reused for a few cycles, which is satisfactory for practical applications.

\section{Acknowledgement}

This works is supported by the Project of the natural science foundation of Jiang Su provincial universities(17KJB220006), and the Priority Academic Program Development of Jiangsu Higher Education Institutions (PAPD).

\section{References}

[1] Du X D, Wang Ch Ch, Liu J G. Extensive and selective adsorption of ZIF-67 towards organic dyes: Performance and mechanism[J]. J. Colloid Interface Sci. 2017, 506:437-441. 
[2] Thai A N, Fu Ch Ch, J R S. Effective removal of sulfur dyes from water by biosorption and subsequent immobilized laccase degradation on crosslinked chitosan beads[J]. Chem. Eng. J. 2016, 304: 313-324.

[3] Pan Y, Wang Y Zh, Zhou A J. Removal of azo dye in an up-flow membrane-less bioelectrochemical system integrated with bio-contact oxidation reactor[J]. Chem. Eng. J. 2017, 326: 454-461.

[4] Decolorization of azo dye by peroxymonosulfate activated by carbon nanotube: Radical versus non-radical mechanism[J]. J. Hazard. Mater. 2016, 320:571-580.

[5] Constantin M, Asmarandei I, Harabagiu V, et al. Removal of anionic dyes from aqueous solutions by an ion-exchanger based on pullulan microspheres[J], Carbohydr. Polym. 2013, 91: 74-84.

[6] Goscianska J, Fathy N A, Aboelenin Reham M M. Adsorption of solophenyl red 3BL polyazo dye onto amine-functionalized mesoporous carbons[J]. J. Colloid Interface Sci. 2017, 505:593-604.

[7] Rasool K, Shahzad A, Lee D S. Exploring the potential of anaerobic sulfate reduction process in treating sulfonated diazo dye: Microbial community analysis using bar-coded pyrosequencing [J]. J. Hazard. Mater. 2016, 318:641-649.

[8] Ozcan Ali, Gencten Metin. Investigation of acid red 88 oxidation in water by means of electro-Fenton method for water purification[J]. Chemosphere. 2016, 146: 245-252.

[9] Yan H, Li H, Yang H. Removal of various cationic dyes from aqueous solutions using a kind of fully biodegradable magnetic composite microsphere[J]. Chem. Eng. J. 2013, 223: 402-411.

[10] Chen R P, Zhang Y L. Wang X Y. Removal of methylene blue from aqueous solution using humic-acid coated magnetic nanoparticles[J]. Desalin. Water Treat. 2015, 55: 539-548.

[11] Carlos L, Cipollone M. Soria D B. The effect of humic acid binding to magnetic nanoparticles on the photogeneration of reactive oxygen species[J], Sep. Purif. Technol. 2012, 91:23-29.

[12] Chen R P, Zhang Y L, Shen L F. Lead (II) and methylene blue removal using a fully biodegradable hydrogel based on starch immobilized humic acid[J]. Chem. Eng. J. 2015, 268: 348-355. 